\title{
Le dessablage des eaux dans les aménagements hydroélectriques
}

\section{Water desanding in hydroelectric schemes}

\author{
PA.R R. MAITRE
}

INGENIEUR AU SERVICE DE IAA PRODUCTYON HYDRAULIQUE E.D.F.

\author{
Mémoire présenté le 28 juin 1957 au Comité technique de la Société Hydrotechnique de France
}

\begin{abstract}
Les ouvrages industriels utilisés pour réaliser le dessablage des eaux sont très divers. On pent cependant dégager parmi eux certaines caractéristiques communes permettant de classer ces ouvrages, d'une part en chambres de décantation it fonctionnement discontinu, d'autre part en dessablenrs propvement dits dont les différents types se ramenent finalement a deux catégories: Les dessablents a écoulement lent o purge répartie le long de l'oubrage, et les dessableurs a écoulement rapide à purge par l'aval. Sur le plan de la theorie da transport en suspension et de la décantation des particules solides. il est de même possible, en précisant les limites de validité des principanx schémas thériques proposés par différents chercheurs, de parventr it une certaine synthese entre des conclusions apparemment discordantes. Toutefois, il reste très difficile de passer de la théorie an calcul pratique des oubrages, qui reste soumis a des règles empiriques approximatives; on pent néanmoins raccorder les différents types d'appareils définis ci-avant anx lois expérimentales de la suspension et du char riage.

La réalisation du dessablage dans les aménagements hydroélectriques modernes pose enfin des problemes particuliers, notamment:

- rentabilité économique du dessablage continu dans les prises a fort degré d'énipement, en particulier pour les cours d'eat glaciaires it sédiments très fins:

- mode d'exploitation optimum des prises i débit solide concentré sur de faibles durées; - dessablage dans les aménagements à prises multiples dont les bussins versants sont hété.rogines.

plusieurs séries de mesures entreprises ces dernières années sur des aménagements existants permettent d'orienter les études dans ce domaine.
\end{abstract}

Nous appellerons dessablage l'extraction des matériaux transportés par suspension à l'entrée des prises d'eau, mais pouvant décanter sous
Industrial plant used for desanding water is very varied. Nevertheless, one can pick out certain charucteristics which all such structures have in common. They can be classed ris intermittent settling out tanks, and desanders proper. The latter fall into two categories: slow flowing desanders with flushing devices located along the length of the structures, and fast flowing desanders with flushing at the dounstream end.

As far as the theory of solid particles being carried along in suspension and settling out is concerned, it is likewise possible, by fixing the limits within which the main theoretical arrangements proposed by various engineers are valid, to arrive at a synthesis of conclusions which are seemingly conflicting. However it is still very difficult to progress from theory to the actual design of structures, which is still governed by approximate empirical laws; it is nevertheless possible to connect the different rpparatus defined above with experimental laws describing suspension and transport.

Desanding in modern hydroelectric schemes sets special problems among which are:

- the economic worth of continuous desanding in offtakes fitted with a large cimount of desanding equipment, especially on glacial. streams and rivers which contain very fine silt;

- the best way of: operating offakes where maximum sediment content occurs over short periods of time:

- desrnding in schemes with more than one intake of which the watersheds are heterogeneous in nature.

The future lines to be followed by research in this field can be determined from several survey programmes which have been carried out on existing schemes in recent years.

l'effet du ralentissement de l'écoulement ou de la diminution de la turbulence.

Il s'agit, en pratique, d'éléments dont le dia- 
mètre varie de quelques centièmes de $\mathrm{mm}$ à quelques millimètres, les éléments inférieurs à quelques centièmes de $\mathrm{mm}$ relevant du délimonage, tandis que les éléments supérieurs à quelques millimètres relèvent du dégravement.

Le dégrav́ement se distingue en outre du dessablage par le lieu où ces opérations peuvent être réalisées dans un aménagement moderne : alors que l'essentiel du dégravement peut être obtenu dans le lit du cours d'eau avant les grilles de prise, le dessablage s'effectue principalement dans une partie des ouvrages d'amenée aménagée à cet effet, sauf dans le cas des retenues de dimensions notables. Quant au délimonage, il n'a lieu que dans les retenues ou réservoirs de grandes dimensions.

CHAPITRE I

\section{OUVRAGES DE DESSABLAGE}

Lc dessablage comporte deux phases :

-.- la décantation des matériaux en suspension,

- leur évacuation à l'extérieur des ouvrages.

Il y' a deux types d'ouvrages :

Chambres de décantation : ouvrages dans lesquels la décantation et l'extraction des dépôts sont des opérations successives.

Dessableur's : ouvrages dans lesquels ces deux opérations sont simultanées.

Le fonctionnement des chambres de décantation implique des interruptions de la marche normale des ouvrages, à la différence de celui des dessableurs.

\section{A) Chambres de décantation}

$1^{\circ}$ Chambres a extraction mécanique:

Nous citons seulement pour mémoire ce type d'ouvrage, aujourd'hui abandonné;

2" Chambres a Évacuation mydrauliove :

Les anciens ouvrages de ce type étaient, en général, à fond plat et comportaient une vanne de chasse de dimensions exiguës, et souvent placée latéralement.

Pour ces trois raisons, l'évacuation des dépôts ne pouvait se faire correctement.

Les ouvrages plus modernes comportent une pente longitudinale de 3 à $5 \%$, parfois une section transversale en trémie, des vannes de chasse de dimensions moyennes $(0,70 \mathrm{~m}$ à $1 \mathrm{~m})$.

Dans certains cas (prise de haute montagne où le bassin de décantation sert également de dégraveur), les sections de vannes de chasse sont beaucoup plus élevées, leur largeur pouvant égaler celle du bassin; les pentes longitudinales, très variables, vont parfois jusqu'à 10 ou $20 \%$. Ces ouvrages simples et rustiques, dont le lonctionnement est en général satisfaisant, présentenl un intérêt particulier pour les petites prises d'eau, et notamment les prises en dessous dont ils peuvent assurer à la fois le dessablage et le dégravement. Ce système comportait jusqu'ici la sujétion de l'intervention humaine pour effectuer les chasses; les premiers résultats obtenus dans les essais de détecteur's d'engravement automatiques permettent d'espérer la disparition de cette sujétion dans un proche avenir.

Toutefois, l'utilisation des chambres de décantation est limitée aux prises de faible ou de

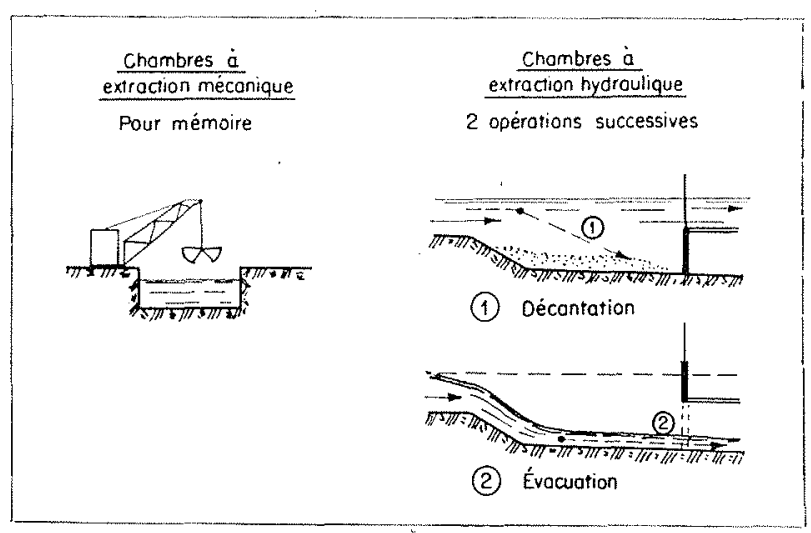

Fig. 1

Chambres de décantation.

* moyenne importance. Il est en effet difficile, sinon impossible, de rejeter brutalement en rivière, lors des chasses, des débits dépassant une dizaine de $\mathrm{m}^{3} / \mathrm{s}$.

\section{B) Dessableurs}

Dans ces ouvrages, il est possible, par le soutirage d'un certain débit liquide, d'extraire les dépôts d'une manière continue durant la marche normale des ouvrages de prises. 
11 convient de distinguer denx catégories de dessableurs en fonction de leur vitesse d'écouloment :

\section{1" LES DESSABLEURS A ECOULEMENT LENT:}

Dans ces ouvrages, la faible vilesse empêche tout charriage de fond sensible. Les matériaux décantés doivent donc être captés, au point nême où ils se sont déposés, par des orifices de purge convenablement répartis.

2. Les Dessableurs a ÉCOUlement rapide:

Dans ces ouvrages, la vitesse est suffisante pour qu'il y ait charriage de fond et il est possible de ne disposer les orifices de purge qu'à lextrémité aval.

\section{Premrère Catégorie :}

DESSABLEURS A ÉCOULEMENT LENT.

a) Généralités.

Vitesse d'écoulement comprise entre 0,20 $\mathrm{m} / \mathrm{s}$ et $0,50 \mathrm{~m} / \mathrm{s}$;

-. Orifices de purge répartis sur toute la longueur du bassin;

Section transversale des bassins en général en forme de trémie.

La faible vitesse d'écoulement, et done la faible turbulence, permettent d'obtenir la décantation des éléments de quelques dixièmes de $\mathrm{mm}$, voire de $1 / 10^{\circ}$ de $\mathrm{mm}$ dans certains cas.

L'absence du charriage de fond permet un certain stockage des dépôts dans les bassins, d'où une souplesse d'exploitation intéressante, l'exploitation pouvant se faire par purge continue ou discontinue.

Toutefois, le nombre élevé d'orifices de purge conduit à une consommation d'eau importante dans le cas de purge continue, et le volume des onvrages conduit à un coût de construction devé.

\section{b) Divers types d'ouvrages.}

1" Dessableur Dufour classique «type 2 .

De nombreux ouvrages de ce type sont en service. Ils ont les caractéristiques communes ciaprès :

-. Pertes d'eau en purge continue : 5 à $15 \%$ du débit dérivé, en général;

- Forme transversale en trémie a forte pente;

- Orifices de purge profilés peu espacés (1 à $2 \mathrm{~m}$ ) et à section décroissante de l'amont vers l'aval;
-.- Vanne de vidange auxiliaire et vannes d'isolement permettant le rinçage des bassins, c'est-à-dire l'exploitation en chambre de décantation;

- Orifice de purge amont de grande dimension pouvant faire orifice de dégraveur.

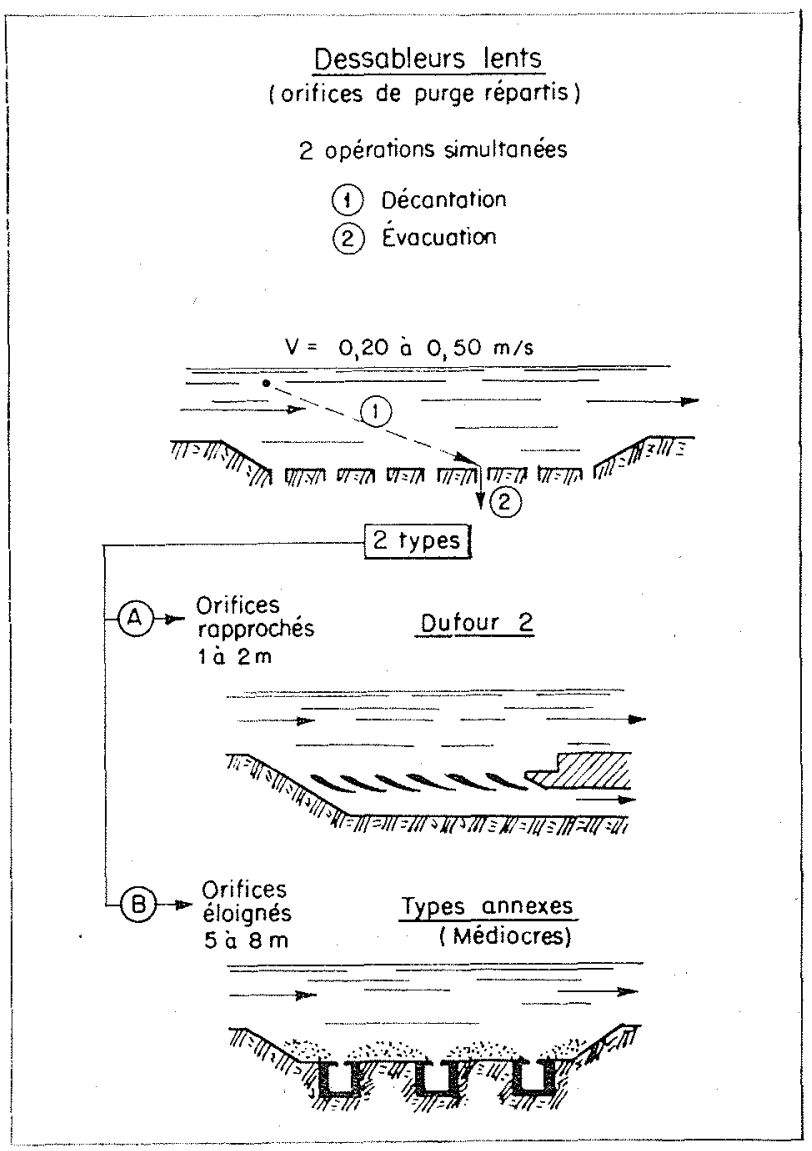

FIG. 2

Dessableurs lents.

2" Autres types (Pont-Escoffier, Pralognan):

On a souvent cherché à réaliser des ouvrages plus économiques que les «Dufour » en diminuant la profondeur des bassins, qui sont à fond plat, et en diminuant le nombre des orifices de purge (espacement 5 à $8 \mathrm{~m}$ ). Les résultats sont, dans l'ensemble, assez médiocres.

On observe en effet les phénomènes suivants : amoncellements de dépôts entre les orifices de purge, éboulements des talus de sable ainsi formés, d'où risque d'obstruction des orifices si ceux-ci sont petits et de remise en suspension d'une partie des dépôts. De plus, le fond plat des ouvrages, calé au niveau du radier des ouvrages d'amenée, et l'absence de vanne d'isolement rendent très difficile le rinçage des bassins et leur exploitation en chambre de décantation. 


\section{Deuxième Catégorie :}

\section{DESSAbLEURS A ÉCOULEMENT RAPIDE.}

\section{a) Généralités.}

Ces ouvrages sont constitués par un canal rectiligne à l'extrémité aval duquel sont disposés quelques orifices de purge.

Les vitesses d'écoulement, notablement plus élevées que dans le cas précédent, doivent à la fois être suffisantes pour que les matériaux déposés soient entraînés par charriage jusque vers l'orifice de purge, et rester cependant inférieures à une certaine limite pour que la décantation puisse se produire. Les vitesses adoptées, en pratique, varient entre $1 \mathrm{~m} / \mathrm{s}$ et $1,50 \mathrm{~m} / \mathrm{s}$, soit le triple environ des vitesses d'écoulement des dessableurs lents.

Ce type de dessableur permet une économie certaine à la construction, les vitesses d'écoulement plus élevées permettant une section transversale plus réduite que précédemment, voire l'utilisation pure et simple du canal d'amenée dans certains cas; ce dernier avantage est assez séduisant lorsqu'on veut placer un dessableur dans un aménagement existant.

La consommation d'eau est, en principe, réduite par le petit nombre d'orifices de purge; elle est, en fait, difficile à chiffrer et semble comprise entre 2 et $10 \%$ du débit dérivé.

Par contre, ces ouvrages ont l'inconvénient de n'avoir un fonctionnement correct que dans une gamme de vitesses limitée: l'existence d'une vitesse limite inférieure restreint les possibilités d'épuration aux sables moyens (de l'ordre de $5 / 10$ de $\mathrm{mm}$ en général, de 2 ou $3 / 10$ de $\mathrm{mm}$ dans les cas très favorables), toute marche à vitesse trop réduite provoquant l'ensablement de l'ouvrage par arrêt du charriage de fond. Le seul mode d'exploitation possible, en marche normale, est la purge continue en raison du charriage de fond permanent; le nettoyage hydraulique des ouvrages par vidange et rincase, c'est-à-dire l'exploitation en bassin de décantation, est impossible en raison de l'absence de vanne de dimensions suffisantes et $d u$ fond plat de ces ouvrages, calé au niveau du radier du canal d'amenée.

\section{b) Divers types d'ouvrages.}

Ces divers types sont caractérisés par la disposition des orifices de purge :

- Orifices frontaux : système « Dufour $3 »$, (cf. fig. $3 \mathrm{~A}$ et 4 );

-- Orifices de fond : système Montagné. - Les orifices sont placés dans un approfondissement du canal en forme de trémie au-dessus duquel est placé un écran horizontal (cf. fig. $3 \mathrm{~B}$ et 5 )
-- Orifices latéraux : système Lévin. - Les orifices sont placés dans la paroi latérale interne d'un coude terminant le dessableur (cf. fig. $3 \mathrm{C}$ et 6 ).

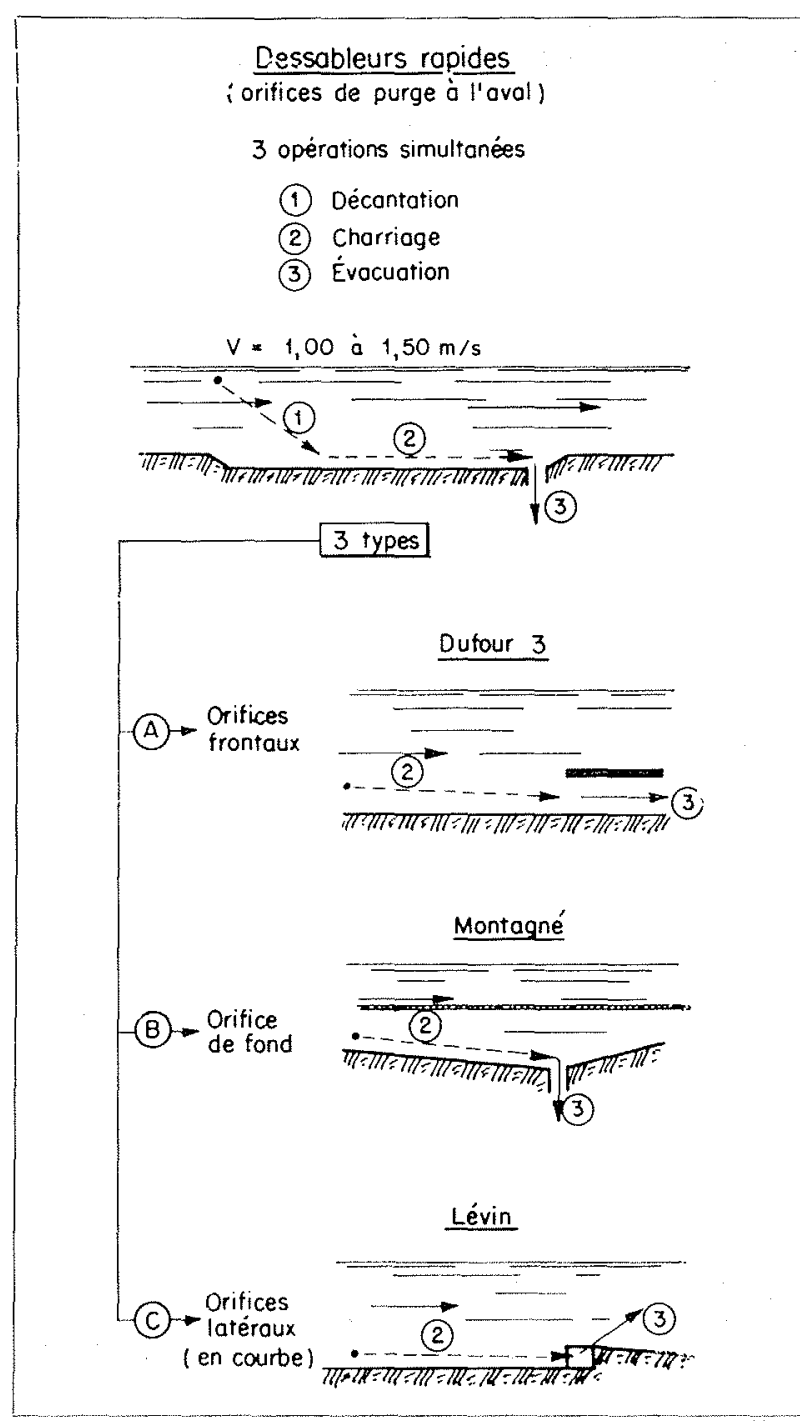

FIG. 3

Dessableurs rapides.

L'utilisation des orifices latéraux reste exceptionnelle; par contre, il serait intéressant de comparer la valeur relative du dispositif à orifices frontaux et de celui à orifices de fond, car l'un et l'autre ont fait l'objet de plusieurs réalisations.

Notons que sur le plan de la décantation proprement dite, les deux systèmes nous paraissent équivalents; on montre aisément, en effet, que, dans le système Montagnné, la présence du divergent formé par l'écran horizontal et le radier ne peut guère améliorer la décantation des parlicules malgré la diminution de vitesse qu'il 
provoque (cf. Nota). Il résulte de ceci que l'addition d'un écran et de trémies Montagné dans la partie aval d'un dessableur à écoulement lent, ne saurait améliorer la décantation; ceci a été
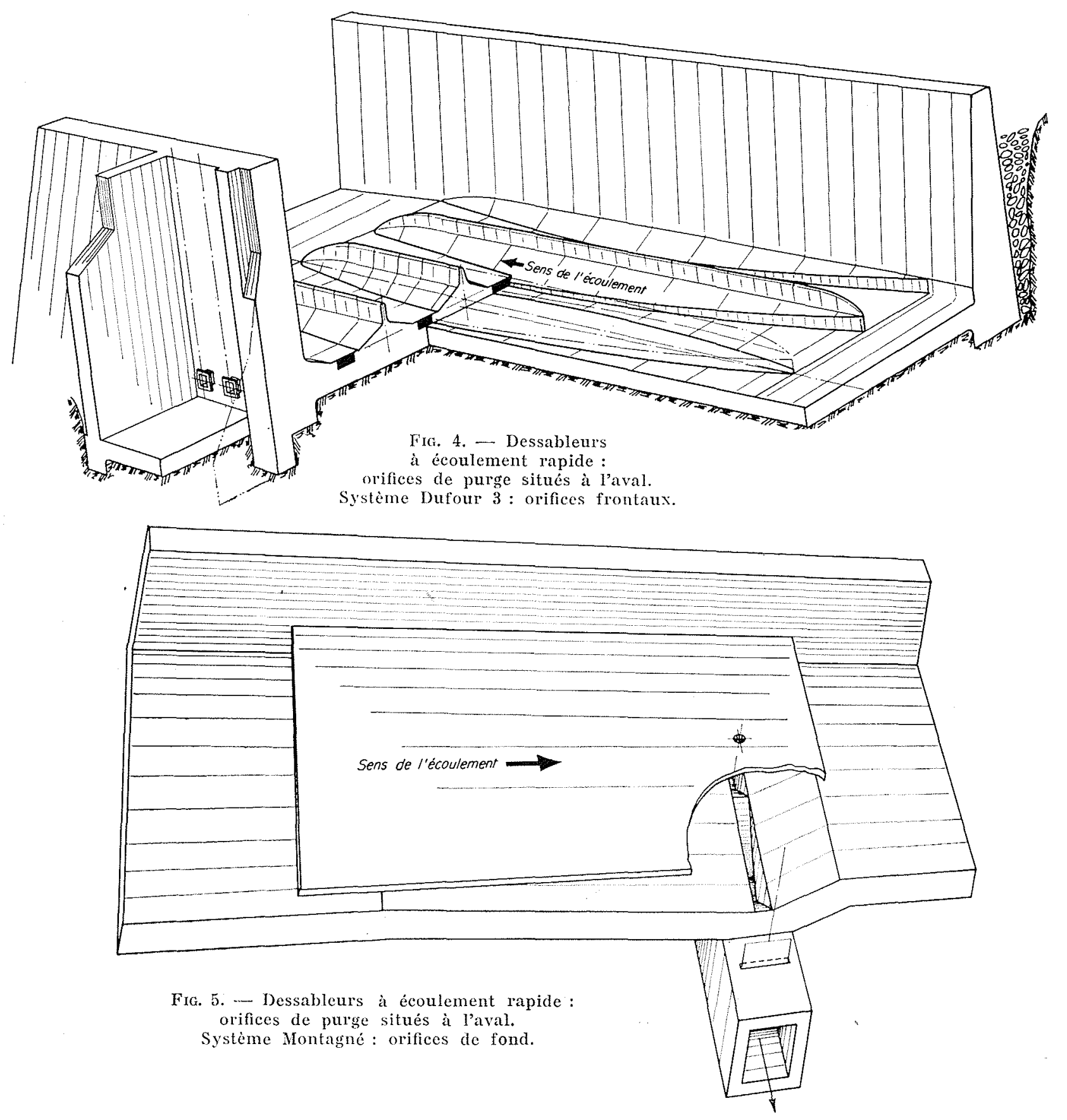

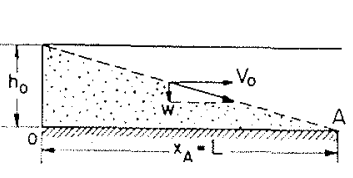

On vérifie que $x_{\mathrm{A}}=x_{\mathrm{A}}$.

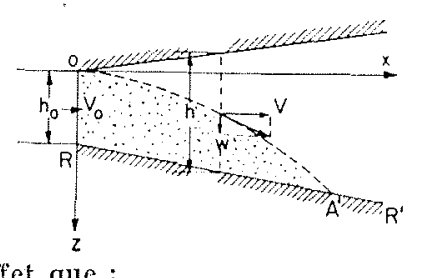

NoTA. - On montre en effet que :

1" Si on néglige la turbulence, la longueur nécessaire à la décantation d'une particule est égale dans un divergent à celle trouvée dans un canal à fond horizontal; dans un canal rectangulaire, cette longueur est d'ailleurs indépendante du tirant d'eau, ce qui explique ce résultat.

$2 \circ \mathrm{Si}$ on tient compte de la turbulence, on constate it la Iumière des récentes études, dont celle de M. Milliat « Etude expérimentale de l'écoulement turbulent dans un conduit divergent $\gg$ (La. Houille Banche, $n^{\circ} \mathrm{B} 1956$ ), que dans un divergent, il n'y a aucune atténuation de la turbulence pouvant favoriser la décantation. 
confirmé par les installations d'essais réalisés à Pralognan et à Pont-Fscoffier il $y$ a quelques années.

Le rôle essentiel de l'écran du système Montagné semble être d'assurer l'évacuation des dépòts à l'abri d'un écran qui sépare la zone des orifices de purge des couches supérieures de l'écoulement, où la vitesse reste élevée. La comparaison du système des orifices frontaux non protégés (Dufour 3) et des orifices de fond protégés (Montagné) nous paraitrait particulièrement intéressante sur le plan pratique de l'exploitation : valeur du débit de purge, efficacité du captage des dépôts, risques d'engorgement. facilités d'entretien... 'Toutefois, elle n'a pu être réalisée à ce jour.

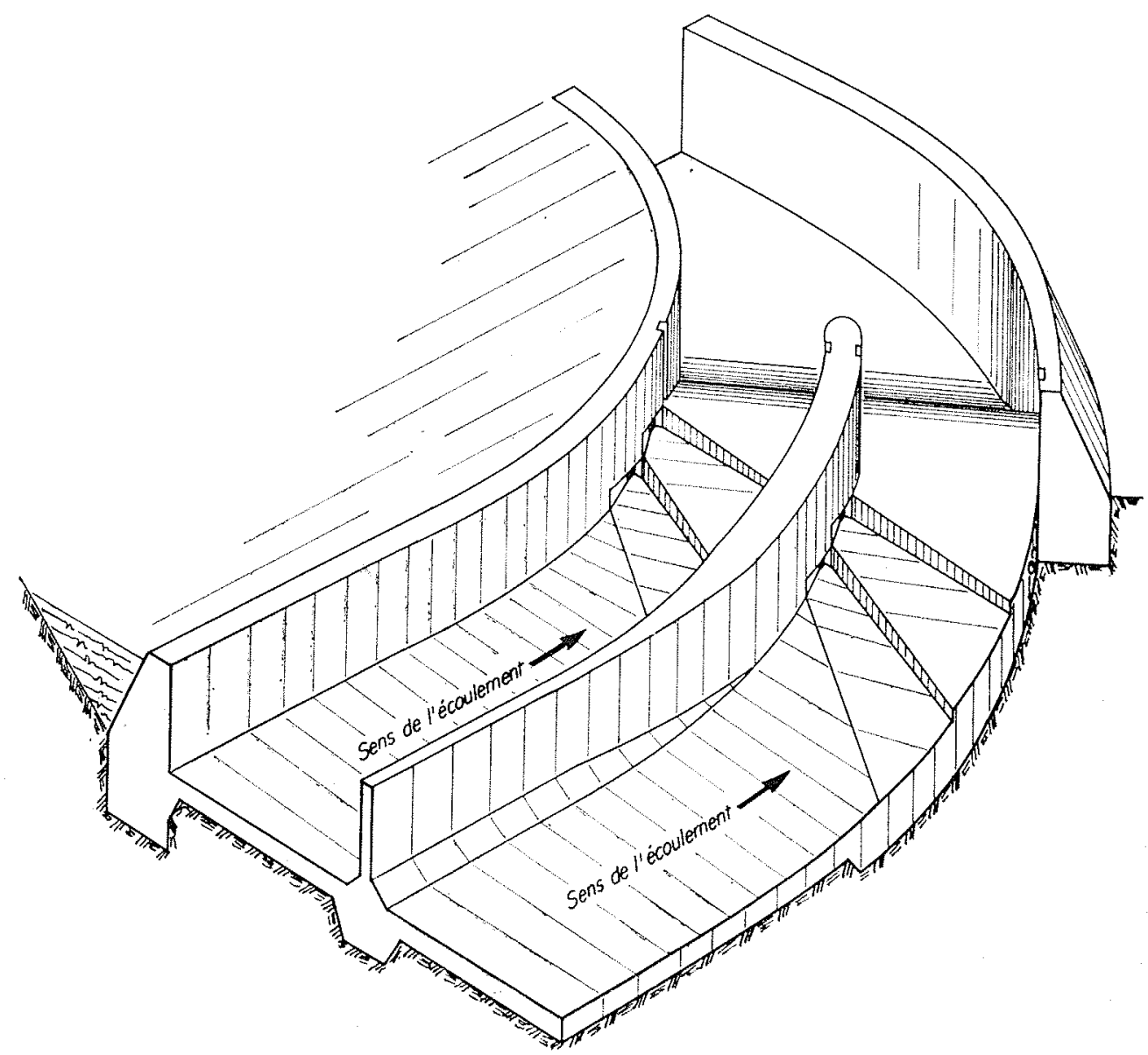

Fig. 6. - Dessableurs à écoulement rapide : orifices de purge situés à l'aval. Système Lévin : orifices latéraux. 


\section{LES LOIS DU TRANSPORT SOLIDE ET LES DIFFÉRENTS TYPES D'OUVRAGES}

\section{A) La décantation des sables \\ en eau courante : Limites de validité des différents schémas théoriques}

Nous analyserons le mécanisme de la décantation des sables pour des vitesses d'écoulement croissantes.

Premer cas. - Vitesse d'écoulement suffisamment faible pour que les fluctuations turbulentes de cette vitesse soient notablement inférieures à la vitesse de chute en eau calme. des grains.

Il est toujours possible, dans ce cas, de donner au dessableur une longueur telle que les grains d’un diamètre donné puissent décanter.

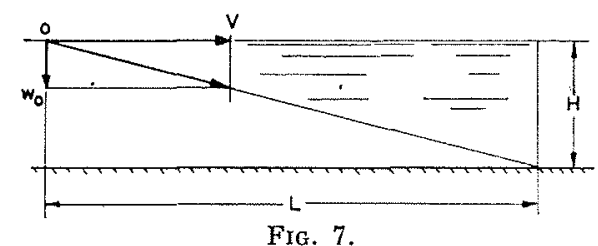

Turbulence nègligeable.

On a en effet la relation élémentaire :

$\frac{\mathrm{L}}{\mathrm{H}} \quad \mathrm{V}\left\{\begin{array}{c}\mathrm{L}=\text { longueur du bassin. } \\ \mathrm{H}=\text { tirant d'eau. } \\ \mathrm{V}=\text { vitesse de l'écoulement supposée } \\ \begin{array}{c}\text { constante. } \\ w_{0}=\text { vitesse de chute en eau calme des } \\ \text { grains. }\end{array}\end{array}\right.$

La relation qui précède s'écrit également :

$$
\mathrm{H} . \mathrm{V}=w_{0} . \mathrm{L}
$$

HV représente le débit par mètre de largeur que peut traiter l'ouvrage pour décanter les grains de vitesse de chute $w_{0}$; ce débit est, on le voit, proportionnel à la longueur du bassin et indépendant de sa profondeur.

DeUXIÉme cas. -- Les fluctuations turbulentes de vitesse ne sont plus négligeables devant la vitesse de chute des grains, qui reste toutefois supérieure à la valeur maximum des fuctuations.

On voit que, dans ce cas, les grains restent nécessairement animés d'un mouvement vers le bas. Cependant, les fluctuations provoquent un freinage de la chute comme l'expérience l'a montré. Ce freinage peut être expliqué en reprenant le schéma proposé par M. Bouvard aux IIes Journées de l'Hydraulique et que nous rappellerons brièvement :

- trainée quadratique $=k w^{2}$;

- vitesse de chute $w$ constante;

- fluctuations de vitesse prenant alternativement la valeur de $\pm v$.

Les deux premières hypothèses reviennent à supposer que les grains sont supérieurs à un certán diamètre, ce qui leur confère un nombre de Reynolds suffisant et une inertie très importante (infinie en théorie).
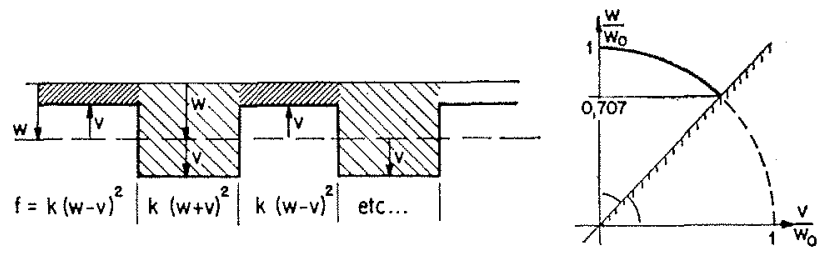

FIG. 8.

Fluctuations turbulentes : $v<w$.

On obtient, après calculs :

$$
\frac{w}{w_{0}}=-\sqrt{1-\cdots \frac{v^{2}}{w_{0}^{2}}} \text { avec }: v<w \text {. }
$$

La relation fondamentale établie dans le cas précédent subsiste en remplacant: $w_{0}$ par $w$, soit :

$$
\frac{\mathrm{L}}{\mathrm{H}}=\frac{\mathrm{V}}{w}=\frac{\mathrm{V}}{w_{0}} \frac{w_{0}}{w}
$$

On voit done que la longueur de décantation $L$ est affectée d'un coefficient de majoration $w_{0} / w$ pouvant atteindre $\sqrt{2}=1,41$, soit $40 \%$. Il est à noter que le résultat précédent correspond à une hypothèse extrême, celle de l'inertie infinie, et que l'hypothèse opposée, celle de l'inertie négligeable, conduit au résultat $w=w_{0}$, les grains étant en glissement relatif permanent à la vitesse $w_{0}$ par rapport à l'écoulement dont les fluctuations vers le haut et vers le bas se compensent.

L'évaluation faite plus haut n'a donc qu'un caractère schématique en raison des hypothèses simplificatrices faites, hypothèses qui s'éloignent 
de la réalité lorsque le diamètre des grains diminue.

M. Bouvard a indiqué que, dans le cas où on écrit l'équation différentielle exacte du mouvement relatif d'une particule sur la verticale, on peut démontrer, dans le cas d'une fluctuation sinusoïdale de vitesse, que la vitesse de chute des particules ne s'annule jamais - le résultat resterait mathématiquement valable pour des fluctuations turbulentes $v$ supérieures à la vitesse de chute $w$. Mais, comme on va le voir, il semble que, dans ce cas, ce soit la mise en équation du phénomène qui soit contestable.

Troisnème cas. -- Les fluctuations turbulentes de la vitesse dépassent, en valeur absolue, la vitesse de chute $w_{0}$ des grains en suspension.

Dans ce cas, le mouvement du grain peut se produire à certains moments du bas ver's le haut; on entre dans le domaine des équilibres de suspension et il n'est plus possible d'étudier le mouvement d'un grain isolément (cf. théorie cinétique des gaz) puisque deux fluctuations de vitesse égales et opposées n'intéresseront pas le même nombre de particules en suspension, à moins que la concentration soit uniforme sur une verticale, ce qui est un cas limite. La notion de trajectoire d'un grain perd son sens et doit être remplacée par la notion d'équilibre des concentrations aux différents niveaux, comme l'ont montré de nombreux auteurs après Schmidt et Rouse. C'est pour cette raison que nous avons limité l'étude de la vitesse de chute en écoulement turbulent d'après M. Bouvard au seul cas où on a : $v<w$. Il convient de remarquer, à l'opposé, que la théorie de Rouse ne correspond à la réalité des phénomènes physiques que si le brassage est effectivement possible; c'est-à-dirc si $v>w$ (ou $v>l w_{0}$ si on suppose l'inertie des grains négligeable, d'où $w=w_{0}$ ).

Or, cette condition passe en général inapercue dans l'exposé de la théorie qui met en évidence le plus souvent deux flux de particules:

- le flux dû à la pesanteur, dirigí vers le bas : $w_{0} \cdot \mathrm{C}$.

- le flux dù au brassage par turbulence, dirigẻ vers le haut : $\varepsilon . d \mathrm{C} / d y$.

Le coefficient $\varepsilon$, produit d'une vitesse $v^{\prime}$ par une longueur de mélange $l$, ne fait pas apparaître le rôle de chacun de ces paramètres; or, on peut concevoir que $\varepsilon$ puisse avoir la même valeur dans deux cas assez différents :

-. soit avec $v^{\prime}$ grand et $l$ petil, d'où un brassage effectif,

- soit avec $v^{\prime}$ petit ef $I$ grand, d'où absence de brassage des particules.
Notons, au passage, l'inconvénient d'un raisonnement trop abstrait dans l'étude d'un phénomène physique, l'équation de Rouse étant souvent établie par analogie avec l'écuation analogue relative au transfert d'une quantité de mouvement ou d'une quantité de chaleur, alors que les conditions de validité sont notablement différentes.

Il y a intérèt, à notre avis, à mettre le phénomène en équation en faisant apparaître, comme l'a fait M. Bouvard, les vitesses résultantes respectives $w_{0}+v$ du flux de particules descendant et $v-w_{0}$ du flux de particules ascendant, soit

$$
\left(v+w_{0}\right) \mathrm{C}_{y_{2}}=\left(v-w_{0}\right) \mathrm{C}_{y_{1}}
$$

Suivant les hypothèses faites sur le schéma de turbulence, on peut, à partir de cette équation, soit retrouver les distributions de Rouse, soit d'autres distributions telle que celle proposée par M. Bouvard.

Si on conserve les hypothèses les plus simplifiées, on retrouve en effet l'équation de Schmidt et Rouse; en posant $y_{2}-y_{1}=l$, il vient (cf (is. 9)

$$
\mathrm{C}_{1 y_{2}}-\mathrm{C}_{1 y_{1}} \# l \cdot \frac{d \mathrm{C}}{d ! J_{\left(y=y_{m}\right)}} \text { et } \frac{\mathrm{C}_{y_{1}}+\mathrm{C}_{y_{n}}}{2} \# \mathrm{C}_{\left(y=y_{m}\right)}
$$

D'où :

$$
\frac{v l}{\varepsilon} \frac{d \mathrm{C}}{d y}+2 w_{0} \mathrm{C}=0
$$

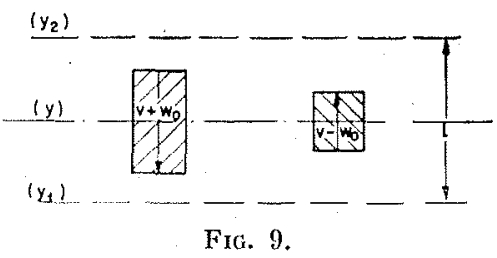

Fluctuations turbulentes : $v>w v_{0}$.

Mais il faut adjoindre à celte équation, pour qu'elle soit valable, la condition $v>w_{0}$ résultant de (1), d'où on déduit que si, à un niveau de l'écoulement, on a $v=w_{0}$, la concentration it l'équilibre doit être nulle en ce point et audessus.

A noter que le coefficient $2 \mathrm{du}$ terme $2 w_{0} \mathrm{C}$ est lié à une anomalie dans la définition usuelle de $s=l^{\prime} \cdot l$.

\section{B) Le calcul pratique des ouvrages de dessablage}

Le fonctionnement pratique des ouvrages se place dans les deux premier's cas examinés au paragraphe précédent, bien qu'en théorie on 
puisse concevoir un dessableur fonctionnant en vogime d'équilibre de suspension et extrayant, le manière continue, la couche de fond la plus chargée en matériaux.

La consommation d'eau d'un tel ouvrage serait prohibitive, compte tenu du résultat d'essais réa-

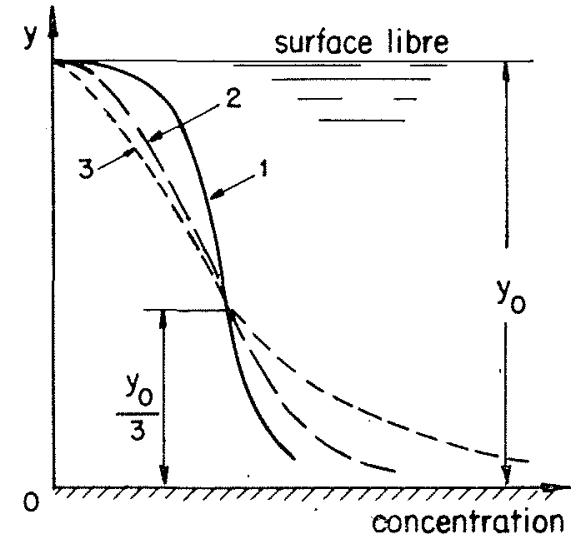

Fig. 10

lisés il y a quelques années au Laboratoire de Chatou et qui ont montré que la déformation des courbes de concentration de Rouse, dans un bassin décanteur, se fait par un pivotement de ces courbes autour d'un point fixe situé à une grande distance du fond (de lordre de $1 / 3$ du tirant d'eau) et non à son voisinage immédiat, comme de nombreux chercheurs l'avaient espéré.

Le calcul des ouvrages de dessablage est donc nécessairement très simpliste, puisqu'il revient à calculer la longueur nécessaire en l'absence de turbulence et à la majorer empiriquement; le coefficient de majoration à uliliser est mal connu : on peut cependant présumer que ce coefficient croît avec le rapport :

$$
\frac{V}{w_{0}}=\frac{\text { vitesse de l'écoulement }}{\text { vitesse de chute des particules }}
$$

Il est à noter, à ce propos, que si la notion de composante verticale de la vitesse ne correspond pas à la réalité des phénomènes physiques, elle pourrait avoir un intérêt pour établir une relation empirique de calcul des dessableurs. On peut écrire, en effet, si on suppose une composante verticale ascendante $V^{\prime}$ de la vitesse, de la forme $\mathrm{V}^{\prime}=\mathrm{AV}$ :

$$
\frac{\mathrm{L}}{\mathrm{H}}=\frac{\mathrm{V}}{w_{0}-\mathrm{AV}}=\frac{\mathrm{V}}{w_{0}} \times \frac{\mathrm{K}}{1-\left(\mathrm{AV} / w_{0}\right)}
$$

On voit que, non seulement le terme correctif $\mathrm{K}$ croît avec $\mathrm{V} / w_{0}$, mais, en outre, il tend vers l'infini pour $\mathrm{V} / w_{0}=1 / \mathrm{A}$, ce qui pourrait établir une correspondance empirique avec la limite de validité de la notion de trajectoire indiquée précédemment.
Les données expérimentales cohérentes nous font actuellement défaut pour chiffrer le coefficient A.

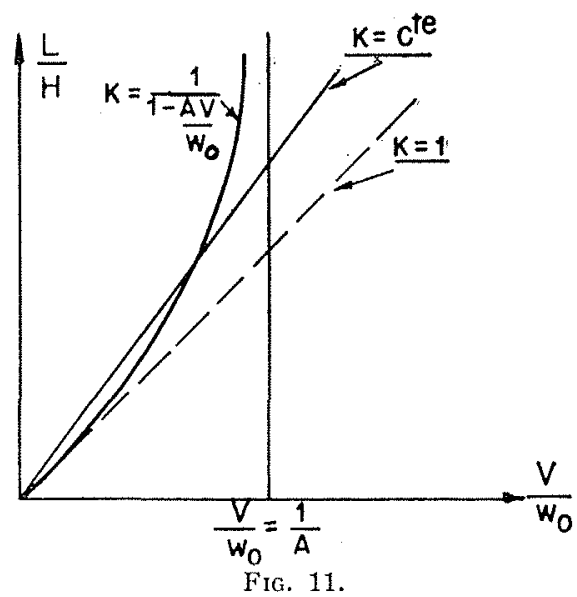

Pour fixer les idées, on peut noter que le coefficient de majoration $\mathrm{K}$ semble voisin de l'unité pour les dessableurs lents $(\mathrm{V}<0,50 \mathrm{~m} / \mathrm{s})$ et les grains de plus de $2 / 10^{\text {es }}$ de mm. Pour les dessableurs rapides, M. Montagné propose de prendre $K=1,5$, sans indiquer de valeur du diamètre de grains. Les essais de dessableur de Lavey semblent conduire à un coefficient de lordre de 4 pour des grains de $1 / 2 \mathrm{~mm}$ et une vitesse de $3 \mathrm{~m} / \mathrm{s}$,

\section{C) Relation entre les lois expérimentales du charriage et de la suspension et le domaine d'utilisation des deux catégories de dessableurs}

On a porté, sur le diagramme figure 12, deux courbes (encadrées chacune par une large zone d'incertitude) représentant, en fonction du diamètre des particules, la vitesse de début d'entraînement sur le fond (A), la vitesse limite de maintien en suspension des grains tendant à décanter (B).

Le tracé A est extrait de Hydroelectric Handbook » de Creager and Justin. Le tracé B, très approximatif, résulte des quelques données expérimentales dont nous disposons.

En considérant les cas limites des particules très fines ou très grossières, on démontre que les courbes $A$ et $B$ se coupent nécessairement.

Si on se déplace sur une verticale du graphique, ce qui revient à augmenter la vitesse de l'écoulement, et si on considère seulement la courbe $B$, on traverse successivement:

_- la zone où la turbulence est négligeable;

- la zone où les fluctuations turbulentes ralentissent la vitesse de chute (traversée de la courbe B); 


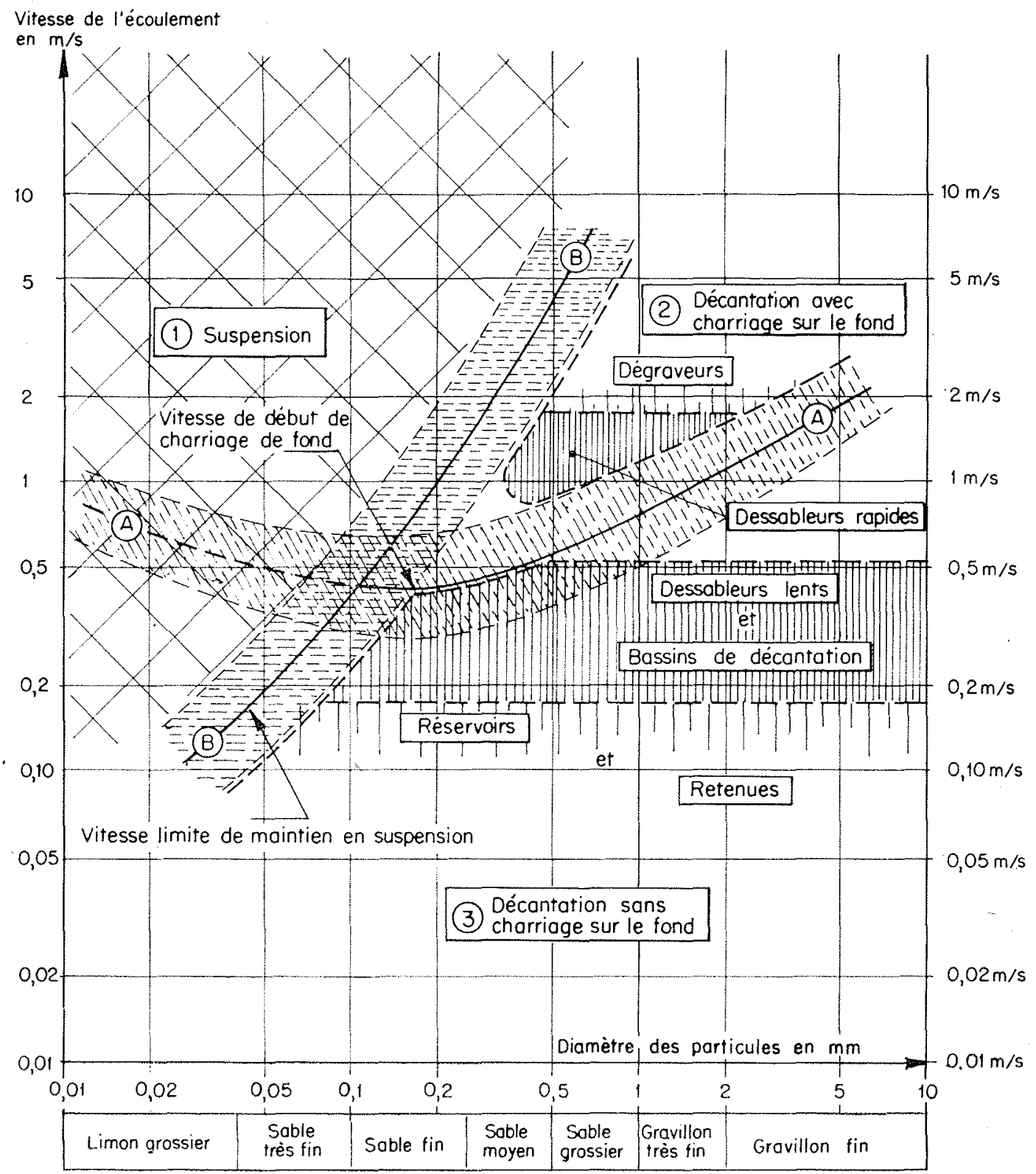

FIG. 12.

- la zone des équilibres de suspension (région 1 du plan).

La zone de turbulence négligeable est subdivisée en deux régions par la courbe A :

la région 2 du plan, dans laquelle le charriage sur le fond est possible, correspond aux dessableurs rapides alors que les dessableurs lents ne peuvent se trouver que dans la région 3 du plan, dans laquelle il n'y a pas de charriage de fond. On remarque combien la zone de fonctionnement correct des dessableurs rapides est réduite, surtout si l'on cherche à faire éliminer à ces ouvrages des éléments assez fins.

\section{D) Conclusion}

Il faut noter :

- Que les schémas d'explication des phénomènes physiques exposés ci-dessus restent souvent grossiers et, par conséquent, en toute rigueur, inexacts;

-.- Que les résultats empiriques chiffrés, présentés ci-dessus, ont été établis à partir d'un nombre très restreint de données expérimentales et peuvent, par là même, nécessiter des corrections sensibles. 


\section{EXAMEN DE QUELQUES PROBLÈMES ACTUELS RELATIFS A CERTAINS AMÉNAGEMENTS MODERNES}

\section{A) Problèmes d'exploitation}

1. L'ÉvOLUTION NÉCESSAIRE DE L'EXPLOITATION DES DESSABLEURS A ÉCOULEMENT LENT DANS CERTIINS AMÉNAGEMENTS MODERNES.

Il s'agit, en général, de dessableurs Dufour classiques.

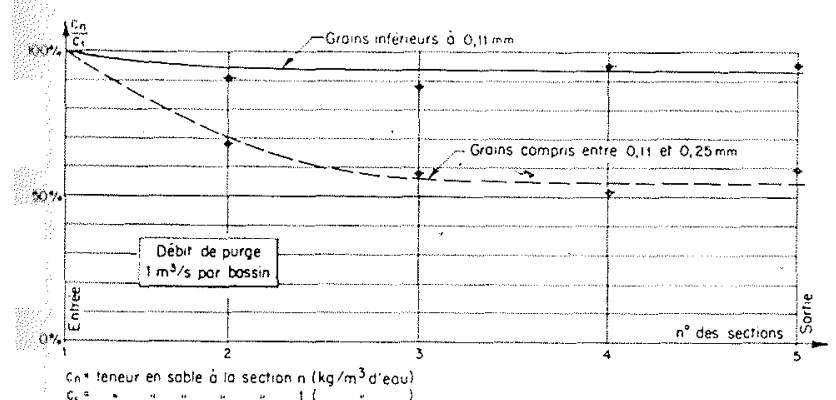

Fugl 13. - Usine de Passs.

Variation de la teneur en sable entere lentre ct la sortie du dessableur.

Ces dessableurs ont été concus à une époquo oì les aménagements étaient caractérisés par un Taible degré d'équipement (d'où excédent de débit disponible en hautes eaux) et par des prises deau dont le dégravement était souvent défeclueux. L'exploitation en purge continue s'imposait donc.

Dans les aménagements modernes, le degré d'équipement est plus élevé, ce qui pose avec rouité le problème de la consommation d'eau; d'autre part, le dégravement est en général assuré dans la retenue.

De plus, dans certains cas de bassins versants glaciaires tels que celui de l'usine de Passy, la finesse des sables restant en suspension à l'entrée du dessableur (95\% de sables inférieurs à $16 / 100^{\circ}$ de $\mathrm{mm}$ ) rend l'efficacité de l'ouvrage très restreinte (cf. fig. 13). Or, dans ce cas particulier, pour éliminer environ $15 \%$ des sables très fins contenus dans l'eau, la consommation d'eau equivaut, certaines années, à une perte de $10 \mathrm{mil}$ lions de $\mathrm{kWh}$.

D'où l'intérêt économique :

- d'exploiter l'ouvrage en bassin de décantation aux périodes de débit moyen, quitte à moins bien dessabler, et à limiter l'exploitation par purge continue aux périodes d'excédent de débit.

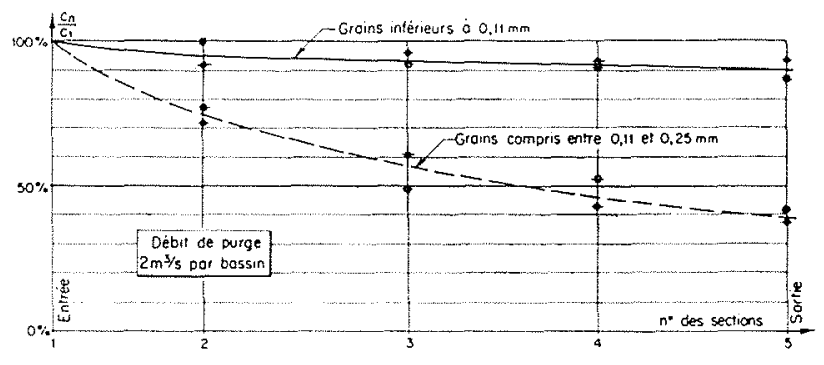

Ce mode d'exploitation, en cours depuis 1956, est complété par des mesures périodiques de rendement des machines afin d'en contrôler la rentabilité. On a déjà pu constater que la composition granulométrique des sables franchissant le dessableur a très peu varié de 1955 à 1956 (cf. fig. 14).

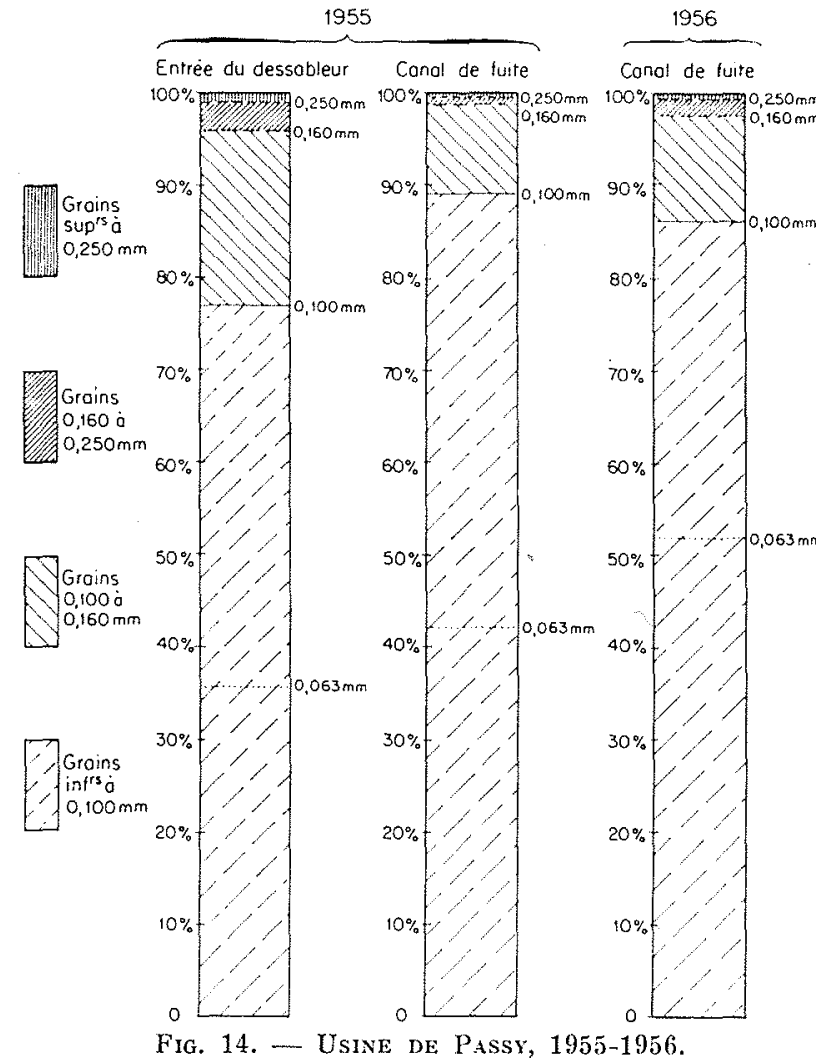

Composition granulométrique moyenne des sables en suspension. 


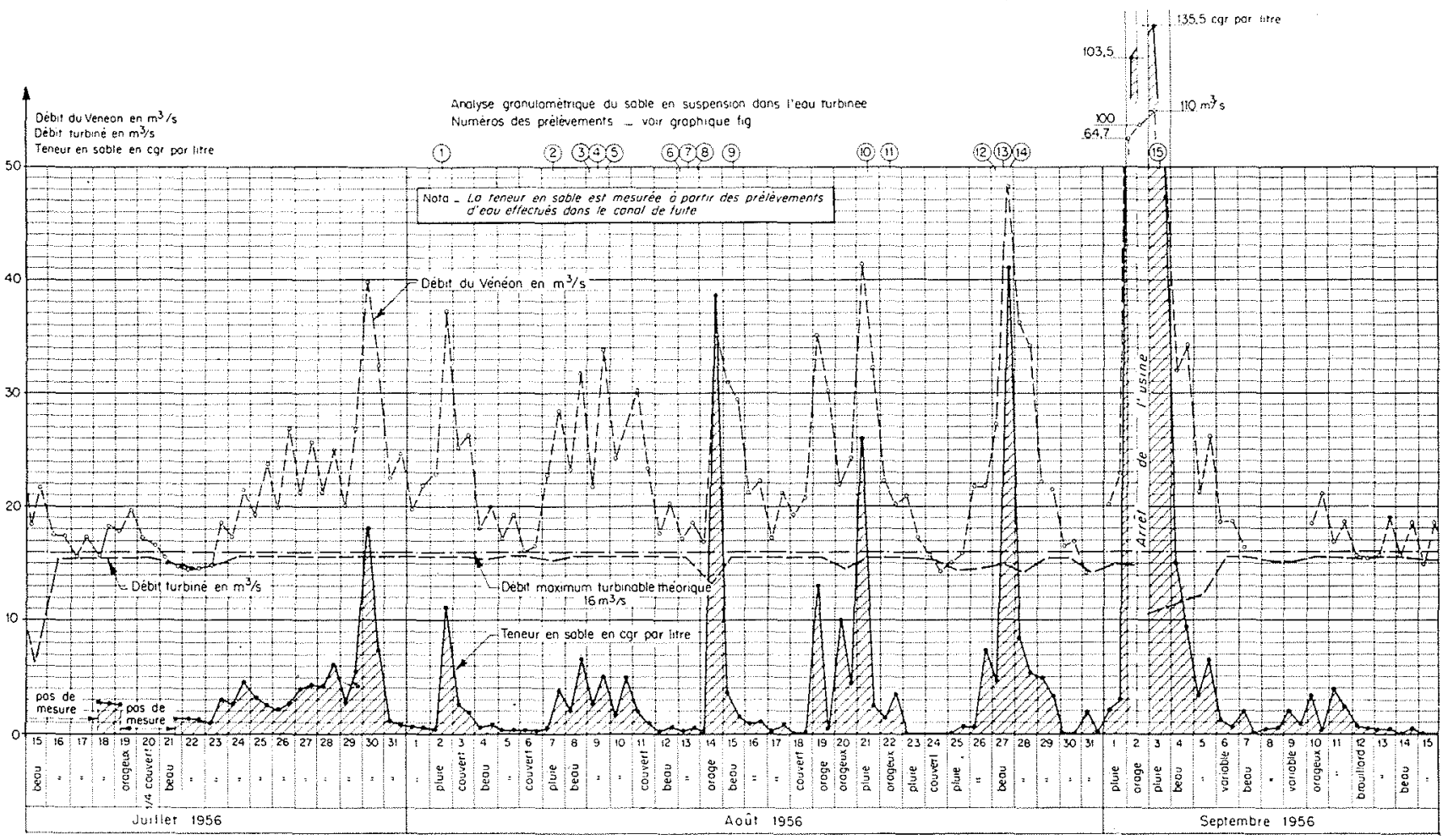

Fig. 15. - Uswe de Pont-Escoffier.

Varialions du débit en rivière et de la teneur en sable de l'eau turbinéc. Année 1956.

$2^{\circ}$ RECHERCHE DE L'EXPLOITATION OPTIMUM DES AMÉNAGEMENTS POUR LESQUELS LE DÉBIT SOLIDF EST CONCENTRÉ SUR UNE COURTE DURÉE.

Les mesures effectuées en 1956 à Pont-Escoffier ont montré que $50 \%$ du débit solide annuel a été turbiné en l'espace de 5 jours environ, lors des crues d'été. De plus, la très forte proportion d'éléments fins ( $85 \%$ de grains inférieurs à $1 / 10^{*}$ de $\mathrm{mm}$ ) rend illusoire toute amélioration du dessabbleur (cf. fig. 15 et fig. 16).

Par contre, on constate que si on réduisait de $50 \%$ le débit turbiné pendant certaines crues, où de nombreux ouvrages déversent, le volume de sable turbiné dans l'année serait réduit d'un tiers, ceci pour une perte d'énergie d'un peu plus de $1 \%$ de la production annuelle. On ne peut cependant conclure sans procéder à un bilan économique complet tenant compte du bilan de production proprement dit et des frais d'entretien dus à l'usure des machines.

Nous espérons que les mesures systématiques de rendement entreprises en 1957 pourront permettre de faire ce bilan, au moins de manière approximative; elles seront conjuguées avec les relevés quotidiens de concentrations en sable, de l'eau turbinée.

Dans l'hypothèse où ce bilan serait favorable, on pourrait envisager d'exploiter certains aménagements à débit réduit pendant quelques jours par an, voire même prévoir des arrêts de

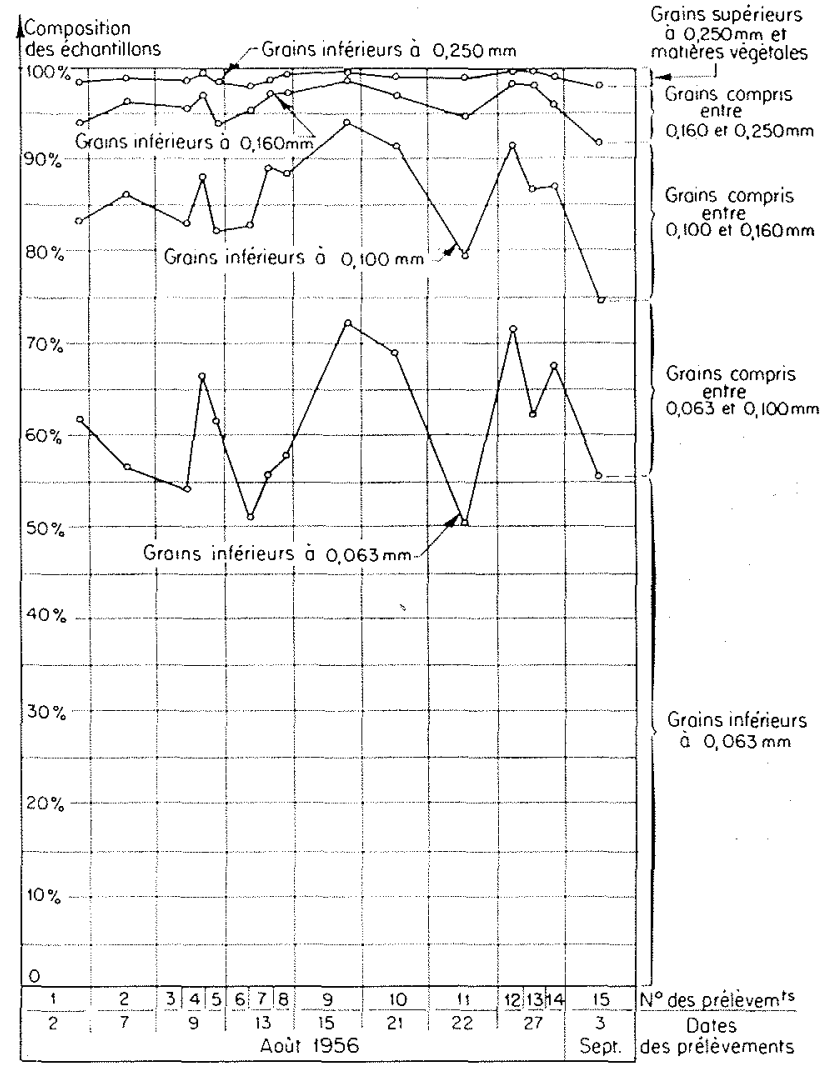

Fig. 16. - Usine de Pont-Escofrier.

Composition granulométrique du sable en suspension dans l'eau turbinée. 
courte durée lors des crues à fort débit solide. 11 y a d'ailleurs lieu de noter que ces diminulions volontaires de production se situeraient pour une large part à des périodes où de nombreux ouvrages déversent.

\section{B) Problèmes d'équipement}

\section{1' INFLUENCE DES DONNÉES NATURELLES:}

\section{a) Gas des prises à prédominance glaciaire:}

Les mesures réalisées ces dernières années aux prises des Houches (usine de Passy), du Plan diu Lac (usine de Pont-Escoffier) et de SaintPierre (usine des Claux), ont mis en évidence une analogie assez frappante des granulométries des sables en suspension à l'entrée des dessableurs, puisqu'on a trouvé une teneur en éléments inférieurs à $0,25 \mathrm{~mm}$ constante $(99 \%)$ pour les trois prises, une teneur en éléments inCérieurs à $0,16 \mathrm{~mm}$ comprise entre 96 et $97,5 \%$, et une teneur en éléments inférieurs à $1 / 10^{\circ}$ de $1 \mathrm{~m}$ variant de 77 à $89,5 \%$. La caractéristique commune de ces trois bassins est la surface de glaciers comprise entre 20 et $25 \%$ du bassin versant.

Il semblerait donc que pour les prises sur bassins glaciaires, il $y$ ait parfois à choisir entre un dessablage extrêmement poussé, de rentabilité parfois discutable, et un dispositif de dégravement largement prévu, pouvant, par sécurité, assurer un dessablage grossier.

\section{b) Cas des aménagements à prises multiples:}

Ce cas, de plus en plus fréquent, fait intervenir, dans un aménagement, des bassins versants de dimension et de nature parfois très différentes. Les mesures réalisées à l'usine d'Aston ont montré par exemple que les sables prélevés à la prise sur l'Ariège, munie d'un dessableur, contiennent $35 \%$ de matériaux rayant l'acier alors que ce pourcentage s'élève à $95 \%$ en moyenne pour les prises secondaires qui ne comportent qu'un dégravement sommaire.

Des constatations du même ordre peuvent être faites sur certains aménagements des Alpes où l'usure des machines est sensible. Citons, par exemple ;

- Malgovert: alimentation mixte à partir d'une retenue et de prises secondaires à dessablage très sommaire et alimentées par des glaciers.

-... Valabres - Bancairon - Courbaisse : prises multiples sur des bassins versants diffé- rents géologiquement et ouvrages de dessablage très inégaux.

Ces exemples montrent l'intérêt d'un examen géologique des bassins versants élémentaires lors de l'étude des différentes prises d'un nouvel aménagement.

$2^{\circ}$ Problèmes concernant le complexe priseDESSABLEUR.

Les points exposés ci-après, pour être moins spécifiques des aménagements modernes que les précédents, nous ont paru néanmoins devoir être mentionnés, au moins brièvement, car de nombreux exemples ont montré que dans le domaine des dessableurs, des défauts de conception apparemment mineurs ont des répercussions importantes sur l'efficacité réelle du dessablage.

\section{a) Alimentation hydraulique :}

Il est certes inutile de rappeler l'importance d'une tranquillisation et d'une répartition transversale des vitesses correcte à l'entrée d'un dessableur. 'Toutefois, il semble qu'en pratique on sous-estime souvent la persistance d'une perturbation quelconque de l'écoulement à l'entrée, en particulier dans les ouvrages à vitesse d'écoulement lent, où les dissymétries d'écoulement ne sont pas apparentes sans mesures, mais n'en perturbent pas moins la décantation.

\section{b) Possibilités de neftoyage des bassins:}

Sur le plan d'exploitation, il est important de pouvoir disposer des moyens d'isolement et de nettoyage des bassins (vannes, seuil aval évitant l'entrainement intempestif des dépôts vers l'usine). Dans plusieurs cas, et notamment à Pralognan, à Pont-Escoffier et à Novaux, l'adjonction de tels seuils a facilité notablement l'exploitation des ouvrages. Il convient de noter ici qu'un des défauts actuels des dessableurs à écoulement rapide, quel que soit leur type, est de se prêter assez mal à l'adjonction de seuils et de vannes de nettoyage de dimensions importantes.

\section{c) Homogénéité de l'ensemble prise-dessableur :}

Chaque fois que le dessableur est au voisinage de la prise d'eau, cet ouvrage doit être conçu et tracé en même temps que la prise afin de s'assurer notamment que les vitesses d'éconlement en hautes eaux sont bien décroissanies de la prise an dessableur, ce qui n'est pas toujours réalisé dans les anciens aménagements (ex. Avrieux).

Notons qu'il convient souvent, dans les ouvrages anciens et même dans certains ouvrages plus 
récents, d'améliorer en premier lieu le dégravement, qui devrait d'ailleurs s'opérer principalement dans la retenue, avant d'aborder le problème plus délicat du dessablage, dont la rentabilité peut même être discutée dans certains cas particuliers, comme nous l'avons vu.

\section{$3^{\circ}$ Dessablage et chorx des machines.}

Est-il utile de rappeler qu'il y aurait intérêt, lors du bilan économique qui préside au choix d'une nouvelle machine, à tenir compte des données sur le débit solide en suspension lorsqu'il s'agit d'éléments très fins et abrasifs? L'usure se manifeste, en effet, de manière différente sui- vant le type de machine choisi : l'entretien des turbines Francis en cas d'usure par le sable est coûteux en raison du nombre de pièces à changer (directrices, labyrinthes, blindages...) alor's que le remplacement des principales pièces d'usure des turbines Pelton (buse et pointeau) est en général plus simple, la roue s'usant à un rythme plus lent. Il y a lieu, par contre, de tenir compte, à l'opposé, de la rapidité de baisse du rendement de chacune des machines en cas d'usure.

Il ne nous appartient pas d'approfondir ici ce dernier problème, qui déborde quelque peu l'objet du présent exposé.

\section{CONCLUSION}

Nous nous sommes efforcé, en premier lieu, de faire la synthèse des travaux de nombreux chercheurs et inventeurs, tant sur le plan des ouvrages industriels de dessablage que sur celui de la théorie de la décantation des sables en suspension dans l'eau, ceci dans le but de permettre au lecteur de situer dans un ensemble tel dispositif ou tel schéma théorique particulier.

Nous espérons, d'autre part, en présentant les études en cours sur quelques cas particuliers, avoir mis en lumière l'évolution rapíde et la diversité des problèmes posés par le dessablage, et par suite la nécessité qui s'impose au projeteur comme à l'exploitant de repenser l'ensemble de cette question dans le cadre de chaque aménagement, compte tenu de l'évolution actuelle des techniques d'équipement et d'exploitation.

\section{IS C US S I O N}

Président: M. HupNen

M. le Président remercie M. Maitre de son exposé très complet et regrette que le retard intervenu dans le déronlement de la séance n'ait pas permis à la communication de M. MaTre d'être suivie par un auditoire beaucoup plus nombreux, qui aurait permis une discussion plus large.

M. Bovvaro estime que si la théorie de la suspension n'est pas à l'heure actuelle utilisable pour le calcul pratique des dessableurs, elle a au moins l'intérêt do justifier qualitativement l'emploi du coefficient de majoration $K$ utilisé par M. MaItre pour le calcul de la longueur d'un dessableur.

M. Montagne précise que, pour les dessableurs rapides (vitesse d'écoulement de $1 \mathrm{~m} / \mathrm{s}$ à $1,50 \mathrm{~m} / \mathrm{s}$ ), la chute des grains de $1 \mathrm{~mm}$ est peu influencée par la turbulence $(\mathbf{K}=1)$, et que la longueur nécessaire à la décantation en l'absence de turbulence est à multiplier par $K=1,3$ pour les grains de $0,5 \mathrm{~mm}$, par $K=2$ pour les grains de 0,25 à $0,3 \mathrm{~mm}$; $K$ devient $\infty$ pour les grains de $0,15 \dot{a} 0,20 \mathrm{~mm}$, ce qui est en accord avec le graphicue (fig. 12) de M. Maitre. De plus un grand nombre de grains de 0,3 ou $0,5 \mathrm{~mm}$ à $1 \mathrm{~mm}$ restent en saltation au-dessus du foud, d'où l'intérêt de ralentir le débit de fond en le captant à quelques décimètres du radier afin de réduire la Iongueur de l'avant-canal.
En réponse à une question posée par M. le Président sur la perte en eau causée par le dessablage, M. Mairre indique que l'eau utilisẻe à cet effet à l'usine de Passy (Haute-Savoie) a représenté une perte en 1955 de $10 \mathrm{mil}$ lions de $\mathrm{kWh}$ sur une production annuelle de $300 \mathrm{mil}-$ lions de $\mathrm{kWh}$. En 1955, la faible hydraulicité de l'année et le fait que l'adduction de la Diosaz n'était pas encore en service ont conduit à prendre toute l'eau nécessaire au fonctionmement du dessableur sur le débit dérivable, ce qui a rendu la perte particulièrement sensible. Mais cette perte est à comparer avec l'économie que procure le dessablage sur l'usure des buses et des roues et sur la baisse de rendement consécutive au turbinage d'eaux chargées de matières solides.

En général, lorsque l'usine comporte une retenue comme à la Girotte, le dessablage se fait dans cette retenue et le problème ne se présente guère qu'en cas de vidange malencontreuse. Cependant, à Aussois, malgrè la présence d'une retenue à l'amont, on a constaté une certaine usure des turbines qui laisserait soupconner, sans qu'on en ait aucune autre preuve, que certaines particules fines resteraient en suspension malgré la vitesse nulle et le séjour de l'eau pendant des mois dans les retenues.

M. Montagné indique que les mesures faites à plusieurs reprises à l'extrémité du divergent formé par la 
fosse creusée sous un écran horizontal ont montré que 1a répartition normale des vitesses conduit à des condilons de décantation qui sont les mêmes que celles des tessableurs lents. Seulement, du fait même d'une bomne décantation, le radier de la fosse doit être creusé en forme de $V$ (en coupe transversale) et son fond muni, tout le long, de trémies à orifices spécialement calcules. Ainsi la consommation d'eau ne dépasse pas de $1 / 40$ à $1 / 20$ du débit du canal et peut être ramenée dans rertains cas à $1 / 80$ de ce débit en réduisant le nombre los trémies de fond.

M. Marrne précise à ce sujet que les mesures effecliées sur le dessableur de l'usine de Campan ont confirmé le fait qu'une décantation complémentaire ne pout se faire sous l'écran horizontal que si le divergent litué à l'entrée est prolongé par un trongon à section 1) a vitesse constantes, ce qui n'est pas le cas dans la whoprt des dessableurs de ce type, en service indusriel it l'heure actuelle. D'autre part, la réalisation d'une licantation plus poussée ne paraît pas compatible avec 11. diminution des consommations d'eau : l'efficacité du lossableur « lent » situé sous l'écran croît avec sa longueur, or le nombre de trémies, et par suite la consommation d'eau, sont également proportionnels a holongueur; le prix de l'ouvrage varie lui aussi dans le nome sens. Si on veut obtenir sune décantation assez poussée, on peut d'ailleurs se demander s'il y a intétot a conserver un dessableur lent sous l'écran horitontal, dans un emplacement peu accessible, ou s'il n'est pas préférable d'adopter la disposition utilisée à Funès col Italie, où M. Dufour a disposé à l'aval d'un dessableur rapide un écran horizontal séparant l'écoulement en deux tranches horizontales superposées, la tranche supélieme étant envoyée directement à l'usine et la tranche niférieure vers un autre dessableur lent, placé à la sortie du premier.

M. Montagne lait remarquer que la consommation deau n'est proportionnelle à la longueur de la fosse que si la profondeur des trémies est invariable. C'est tinsi que pour des trémies normales et un débit de burge, également normal, de $1 / 20$ à $1 / 30$ du débit du canal, une plus srande profondeur des trémies (trèss ncceptable) conduit, pour une plus grande longueur de cosse, a réduire leur nombre, done celui des orifices et 1) faire passer le débit de purge à $1 / 40$ seulement.

M. Martre répond que, dans ces conditions, la diminution du débit de purge n'est obtenue qu'en sacrifiant 1. caractère économique de ce type de dessableur, essentiellement lié à sa faible profondeur: Ia présence de lrémies $p \triangleq u$ nombreuses, donc très profondes, augmente en effet notablement le contt du génie civil de l'ouvrage.

M. Bouvaro estime que le rôle important attribué jusqu'ici au dessableur semble justifie dans une cerlaine mesure par quelques résultats expérimentaux :

- a Pont-Escoffier : l'usure des turbines a diminué sensiblement au fur et à mesure que le dessableur se perfectionnait : ce dernier ne laisse guère passer actuellement que des grains inférieurs au $1 / 10$ de $\mathrm{mm}$; l'amélioration des métaux utilisés pour les turbines no suffit pas, semble-t-il, à expliquer la diminution d'usure;

- à Passy : les problèmes posés par l'usure sont beaucoup moindres qu'à l'usine de Chedde située sul' le même troncon de l'Arve avec une chute moitié plus faible que Passy dont la prise ne possede ni dessableur ni possibilités de chasses.

M. Marrae pense que l'utilité d'un dessableur doit se juger, à sa construction, sur le plan de la rentabilité economique, et non sur les améliorations d'un ouvrage existant. En effet, si la colrection des défauts présentés initialement par le dessableur de Pont-Escoffier (fond plat, orifices trop petits et trop espacés) a permis d'améliorer son efficacité, très faible à l'origine, il n'en résulte pas immédiatement que la construction de cet ouvrage serait rentable si on l'envisageait actuellement alors que, avant dessablage, plus de $80 \%$ des sables en suspension sont inférieurs au $1 / 10 \mathrm{~mm}$. On pourrait se poser la même question au sujet de l'usine de Passy dans laquelle les essais en cours semblent montrer que les machines sont peu sensibles à une réduction du dessablage: la comparaison avec l'usine de Chedde peut d'ailleurs être contestée, les caractéristiques de la prise d'eau elle-même et des machines n'étant guère comparables

Les différentes phases du dessablage et de la decantation, avec extraction mécanique et extraction hydraulique, suggèrent à M. le Président un parallèle avec l'evolution des filtres utilisés dans les adductions d'eau, où l'on a commencé par laisser les filtres s'engorger, le nettoyage étant fait a la main; ensuite on a fait du nettoyage hydraulique en inversant le courant. Une communication sur ce parallèle, faite en collaboration avec la Section Filtration, Infiltration et Eaux souterwaines, serait souhaitable dans le champ d'activité de la S.H.F.

M. Marrre rappelle que, dans le domaine des équipements hydrólectriques, il semble qu'on revient plutot a l'utilisation de chambres de décantation (ouvrages i fonctionnement discontinu) pour éviter les pertes d'eau. Sur les prises de haute montagne on commence actuellement à automatiser les vannes des chambres de décantation, dont l'ouverture nécessitait jusqu'ici une présence humaine, ce qui conduisait souvent les projeteurs à préroir des dessableurs continus pour éviter cette sujétion.

N. Bouvaro confirme que si on définit le rendement d'un dessableur par le nombre de $\mathrm{m}^{3}$ d'eau qu'il faut utiliser pour évacuer un mètre cube de matériaux de granulométrie donnée, on constate assez aisément que c'est le dessableur à purge discontinue qui est de beaucoup supéricur à ce point de vue là. Ce serait à son avis une raison suffisante pour justifier l'installation dans les bassins de dessablage continu, par exemple type Dufour, d'une vanne de vidange beaucoup plus importante que celle habituellement prévue permettant de faire des purges discontinues dans de meilleures conditions. C'est cette solution qui a été utilisée à la prise de Diosaz.

Il faut par ailleurs s'attendre à ce que les dessableurs soient des ouvrages dont le cont croît beaucoup plus vite que l'efficacité.

M. Bonnin indique, à propos de la perte annuelle de 10 millions de kWh par dessablage, que les frais de remise en état des turbines Pelton de l'usine de Pont Escoffier où il $y$ a une production du même ordre qu'à Passy, étaient aux environs de 1950 de lordre de 30 à 50 millions de franes.

M. Mattre remarque que les depenses et la perte d'énergie en kWh sont sensiblement du même ordre en valeur absolue. If propose à $M$. Bonvis de revoir cette question avec lui, compte tenu des progrès constants des techniques d'entretien des roues et du fait que, même en dessablant, on continue à garder des particules fines, de telle solte qu'il faut comparer les frais de dessablage non avec les frais totaux d'entretien, mais avec la diminution des frais que le dessablage permet de réaliser : à Passy, où l'on n'enlève que 10 à $15 \%$ des matériaux, cette diminution, des frais serait, a partir des chiffres indiqués par M. BonNw, de l'ordre de 5 millions, alors que la perte en eau correspondante représente $30 \mathrm{mil}-$ lions de francs. 